\title{
Dynamic Characteristics Estimation of Cable-Stayed Bridge Using Artificial Filter Bank (AFB)
}

\author{
Gwanghee Heo and Joonryong Jeon
}

\begin{abstract}
This paper aims at determining dynamic characteristics of cable-stayed bridge by means of an artificial filter bank (AFB) for the purpose of efficient attainment of valid dynamic responses in real-time. The AFB was developed by using a band-pass filter optimizing algorithm and a peak-picking algorithm, and it was embedded into a wireless sensing system. Then a modal testing was performed on a cable-stayed bridge model and its natural frequency was calculated to capture dynamic characteristics. They were evaluated in comparison to responses from wired measurement system and FE analysis results for validation. Finally, the AFB based dynamic wireless measurement system was validated for obtaining effective dynamic (acceleration) responses compressed within the frequency range of interest with technological and economical efficacy.
\end{abstract}

Index Terms-Artificial filter bank, band-pass filter optimizing algorithm, peak-picking algorithm, construction error, compressive ratio.

\section{INTRODUCTION}

For structural health monitoring (SHM) of civil infrastructures, it is important to effectively measure valid structural responses in real-time [1]-[5]. Structural responses were traditionally measured via separate static/dynamic data loggers with wires, but recently new ways of measurement are being studied utilizing wireless sensor networks (WSNs) that integrate rapidly developing information technologies and radio frequency (RF) communication technologies. Although WSNs, according to these studies, display weaknesses in power consumption, efficiency, lack of robustness of wireless network, and some more, it is expected to grow more in applications with continued development due to its strengths in the mobility of wireless system, easily installable sensors, and low cost of system configuration and maintenance [6]. Dynamic response of a structure using WSNs will contain more data compared to that of the static response, and may result in data loss due to bottleneck during wireless transmission of the dynamic data and also cost increase from integrating and managing large database (DB) accordingly. Therefore, in order to obtain and manage dynamic responses of infrastructure using WSNs in real time with reliability, a technical alternative needs to be proposed to filter valid dynamic responses so as to efficiently obtain and transmit only necessary data [7].

In this study, an artificial filter bank (AFB) is developed

Manuscript received March 20, 2014; revised May 28, 2014.

The authors are with Department of Civil and Environmental Engineering, Konyang University, 119 Daehangro, Nonsan 320-711, South Korea (e-mail: heo@kyu.ac.kr, jrjeon@kyu.ac.kr). using band-pass filter optimizing algorithm and peak-picking algorithm, and embedded into a wireless measurement system in order to filter and obtain effective dynamic response, or acceleration response of infrastructure in real time. Also a modal test was performed on a model of cable-stayed bridge to analyze dynamic characteristics while wired measurement and finite element (FE) analysis were conducted in parallel for comparison and evaluation. Finally, the AFB developed in this study was able to obtain effective dynamic response compressed around frequency range of interest via WSNs in real time, achieving economic and technological efficacy, and providing a new paradigm in real-time measurement, transmission, and management of dynamic structural responses to analyze large infrastructures and other structures.

\section{ARtificial Filter BAN-BASED DyNAMIC WirELESS MEASUREMENT SYSTEM}

\section{A. Artificial Filter Bank}

An artificial filter bank (AFB) was developed on the basis of band-pass filter optimizing algorithm and peak-picking algorithm to efficiently collect dynamic response data of infrastructure. First, a band-pass filter optimizing algorithm is designed in parallel with multiple band-pass filters making it appropriate for determination of the target mode required for evaluation of dynamic states where the number, bandwidth, spacing and other design parameters of band-pass filters are repeatedly modified for computing a reconstruction signal. Finally such a reconstruction is evaluated by comparing the raw data attained from target structure to the reconstruction signals while the frequency range of interest is set below $10 \mathrm{~Hz}$ to consider the dynamic characteristics of large scale infrastructures. To evaluate the effects of reconstruction signals, reconstruction error (RE) is used as seen in (1).

$$
R E=\frac{\int_{0}^{T}|u(t)-y(t)| /|u(t)|}{T}
$$

where $u(t)$ is the raw data of response in time domain, $\mathrm{y}(\mathrm{t})$ is the reconstruction signal of response in time domain, and $\mathrm{T}$ is the total period of input signal. The closer RE is to zero, the more effective reconstruction becomes. At this point, Lynch [7] introduced a statistical concept for determination of reconstruction error, applying a residual sum of squares (RSS). Yet, this method offers only a contingent criterion which reveals variations of reconstructed signals in contrast to original ones. A further clarification about how reconstruction is effective requires a criterion which would 
be universally applicable by dividing the difference between original and reconstructed signals by original ones, as suggested in (1).

Next, peak-picking algorithm is developed to re-sample peak values based on time information of only peak values picked from reconstruction signal determined by (1), where peak value is determined only when there is a sign change while calculating the slope of each sample signal. Finally, resulting compressive samples are transmitted via WSNs to a data administrator, and compressive ratio (CR) (2) is used to evaluate compressive efficiency of the re-determined compressive sample from the reconstruction signal.

$$
C R=\frac{N S_{C}}{N S_{O}}
$$

where, $N S_{C}$ is the number of compressive samples from peak-picking signal, while $N S_{O}$ is the number of samples from reconstruction signal. When the CR gets close to zero, the compressive efficiency increases. The AFB combining these band-pass filter optimizing algorithm and peak-picking algorithm is programmed using Matlab to make it embedded in a dynamic wireless management system (DWMS) as in Section B.

\section{B. Dynamic Wireless Measurement System}

In this study we set up a dynamic wireless measurement system (DWMS) following the design of digital software based on a real-time operating system, for completion of the AFB embedded system developed in Section A and for modifiability and applicability of band-pass filter in the future. Of system components, logging and controller use cDAQ-9139 of National Instruments (NI), which carries linux based RTOS. If software based design of function and logics of previously developed AFB get embedded, those function and logics can be recognized and executed via auto code generation. Next, dynamic (acceleration) responses are measured using NI-9233, which supports piezo-electric accelerometers and is made of four channels with expandable channels in case of additional connections. For two-way RF communication, AWK-3121 module from Moxa was used, and this module has bandwidth of about 11 54 Mbps with $100 \mathrm{~m}$ transmission range for real-time RF communication between cDAQ-9131 and administrator PC. The administrator $\mathrm{PC}$, or the main control system, is equipped with graphic user interface (GUI) via Labview to acquire, analyze, and store the transmitted acceleration responses from multi-channels in real-time.

\section{FE ANAlysis AND Model Test of CABLE-Stayed BRIDGE MODEL}

\section{A. FE Analysis of Cable-Stayed Bridge Model}

Before testing the characteristics of cable-stayed bridge model, this study analyzed it for eigenvalues using FE modeling. The bridge model looks as in Fig. 1, and dimensions of the bridge model are as in Table I. The FE analysis of the model bridge was performed using commercial structural FE analysis program, I-DEAS of UGS, while girder and tower are assumed 1D beam element, cables assumed spring element, and boundary conditions of towers and bridge ends assumed as in Table I for FE modeling. The Fig. 2 shows the completed FE model, and eigenvalue analysis results for first to third bending modes are tabulated in Table I.

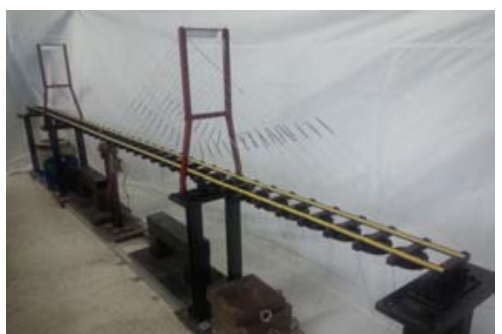

Fig. 1. View of cable-stayed bridge model.

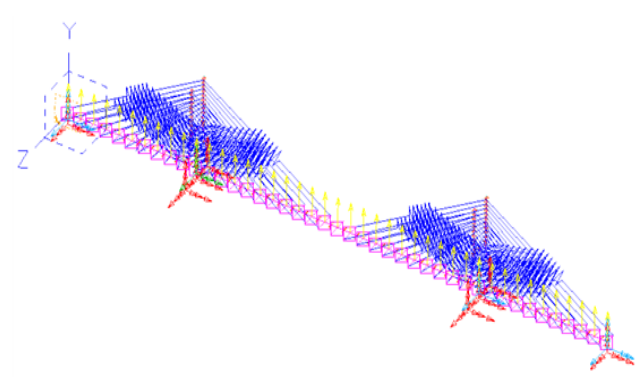

Fig. 2. FE Model of cable-stayed bridge model.

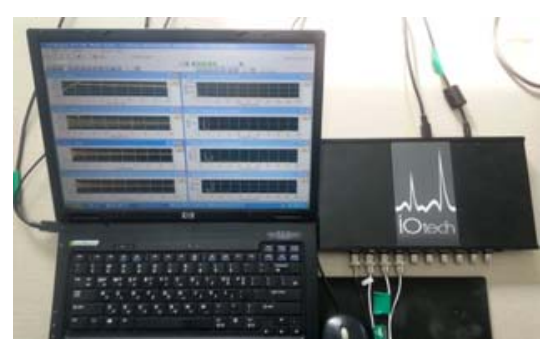

Fig. 3. Dynamic wired measurement system.

TABLE I: DESIGN DIMENSIONS OF CABLE-STAYED BRIDGE MODEI

\begin{tabular}{c|c}
\hline \hline Category & Values \\
\hline Total/main span lengths & $4.22 \mathrm{~m} / 2.22 \mathrm{~m}$ \\
\hline Superstructure width / Tower heights & $0.17 \mathrm{~m} / 1.00 \mathrm{~m}$ \\
\hline Tower boundary conditions & Roller and hinge \\
\hline Bridge end boundary conditions & Roller \\
\hline Bridge materials & Structural steel \\
\hline Cable materials & Spring $(\mathrm{K}=1.03 \mathrm{~N} / \mathrm{mm})$ \\
\hline Concentrated loads & $1 \mathrm{kgf}(39 \mathrm{EA})$ \\
\hline \hline
\end{tabular}

TABLE II: EIGENVALUE RESUltS OF FE MODEL

\begin{tabular}{c|c|c}
\hline & \multicolumn{2}{|c}{ Bending modes } \\
\hline $1^{\text {st }}$ & $2.877 \mathrm{~Hz}$ & \\
\hline $2^{\text {nd }}$ & $4.194 \mathrm{~Hz}$ & \\
\hline $3^{\text {rd }}$ & $6.242 \mathrm{~Hz}$ & \\
\hline \hline
\end{tabular}

\section{B. Modal Test of Cable-Stayed Bridge Model}

A modal test of cable-stayed bridge model was performed to evaluate validation of the dynamic wireless measurement system which will be used for AFB. The Modal test was conducted by wired sensor system and DWMS. Acceleration 
response in vertical direction for both the measurement systems was taken from $1 / 3$ of the main span on the girder where all 3 bending modes can be measured concurrently. Fig. 3 and Fig. 4 show the dynamic wired and wireless measurement system respectively. The wired dynamic measurement system used is iO-tech (Model: 652U) and ME'Scope from Vibrant Tech.

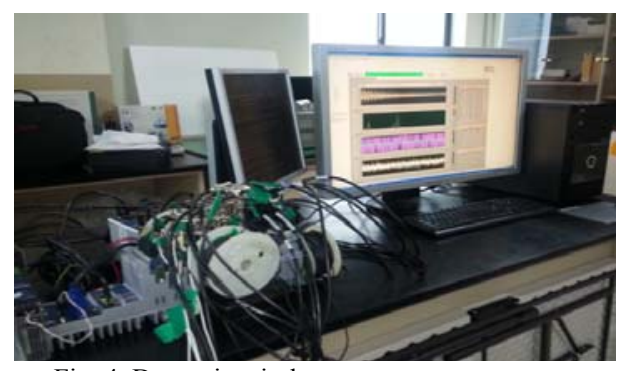

Fig. 4. Dynamic wireless measurement system.

\section{Results of FE Analysis and Model Test}

Fig. 5 and Fig. 6 show the measured raw data from wired and the dynamic wireless measurement system in both time domain and frequency domain while Table III summarizes the natural frequency of both data as well as the FE analysis result from Section B (Part II). Just as for FE analysis results, eigenvalues for three bending modes are calculated, and the accuracy of FE model and the validity of the dynamic wireless measurement system configured in this study are evaluated via error calculation based on the wired dynamic response values.

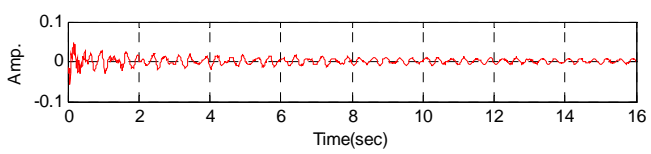

(a). Time response.

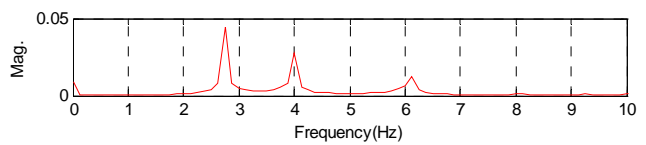

(b). Frequency response.

Fig. 5. Response of wired dynamic measurement system

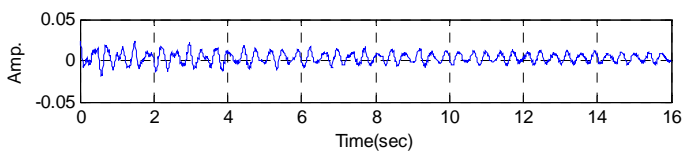

(c). Time response.

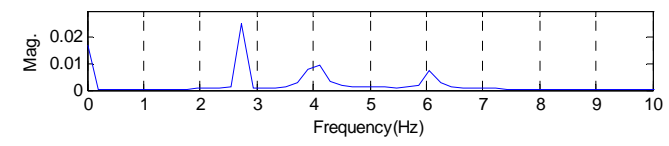

(d). Frequency response.

Fig. 6. Response of wireless dynamic measurement system.

TABLE III: COMPARE TO EIGENVALUE OF FE ANALYSIS AND MODEL TEST

\begin{tabular}{c|c|c|c|c|c}
\hline \hline Bending & $\begin{array}{c}\text { Wired } \\
(\mathrm{Hz})\end{array}$ & $\begin{array}{c}\text { FE anal. } \\
(\mathrm{Hz})\end{array}$ & $\begin{array}{c}\text { Error } \\
(\%)\end{array}$ & $\begin{array}{c}\text { Wireless } \\
(\mathrm{Hz})\end{array}$ & $\begin{array}{c}\text { Error } \\
(\%)\end{array}$ \\
\hline $1^{\text {st }}$ & 2.735 & 2.877 & 5.191 & 2.736 & 0.036 \\
\hline $2^{\text {nd }}$ & 4.005 & 4.194 & 4.719 & 4.104 & 2.471 \\
\hline $3^{\text {rd }}$ & 6.154 & 6.242 & 1.429 & 6.058 & 1.559 \\
\hline \hline
\end{tabular}

The eigenvalues of $\mathrm{FE}$ analysis results in comparison to the wired values showed error of about 1.429 5.191\%, which is quite close to the wired value, showing that FE model in Section B (Part II) significantly simulates the dynamic characteristics of model bridge. The dynamic wireless measurement system results are only showing error of $0.036 \sim 2.471 \%$ compared to the wired results, confirming the validity of the wireless measurement system.

\section{Performance Evaluation of AFB}

The AFB embedded in the dynamic wireless measurement system was designed with 6 band-pass filters, $0.6 \mathrm{~Hz}$ bandwidth, and $1.0 \mathrm{~Hz}$ of filter spacing. The specified design parameters are optimum for obtaining structural response under $10 \mathrm{~Hz}$ when the famous E1-centro random wave is applied to a structure. Because structural response of infrastructures essentially contains random characteristics in usual accidental events (earthquake, wind gust, shock), this study assumed more realistic measurement situation and focused on structural response attainment to select a target mode. Reconstruction signal and data compression ratio are used to evaluate performance of the AFB. Reconstruction signal is computed and compared to the original signal of the dynamic wireless measurement system in Fig. 7.

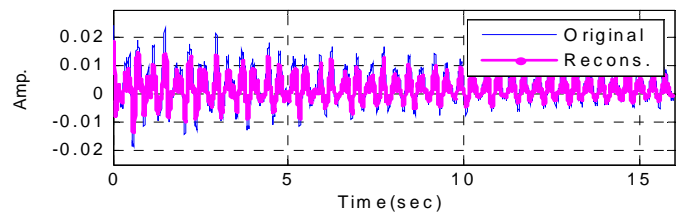

(a). Time response.

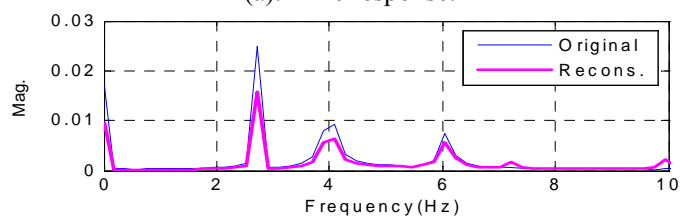

(b). Frequency response.

Fig. 7. Compare to original and reconstruction signal.

It can be seen that reconstruction signal sufficiently contains and reconstructs modal values of the original signal, proving that band-pass filter optimizing algorithm of AFB is effective in vigorously highlighting and illustrating target (interest) mode within design conditions of $10 \mathrm{~Hz}$ bandwidth.

Next, this study designed and applied peak-picking algorithm to the AFB developed for data compression for efficient operations and management of RF communications and measurement DBs. The peak-only signals are computed and compared to the reconstruction and the original ones in time domain and frequency domain in Fig. 8. Fig. 8(a) shows that only peak values of reconstruction signal are extracted using the peak-picking algorithm, and Fig. 8(b) shows that peak value-only signals definitely include the modal information accordingly. In conclusion, the peak-picking algorithm of AFB is effective in extracting peak values containing modal information of the entire reconstruction signal.

For a quantitative evaluation of AFB, this study tabulates eigenvalues of the reconstruction signals and compressive ones and their errors relative to the original signals as in Table IV. Also the reconstruction error (RE) comparing reconstruction signal to the original one, and the compressive 
ratio (CR) comparing compressive signal to the reconstruction signal are calculated in Table V. Table IV shows that reconstruction signal shows $0 \%$ error in eigenvalues compared to the original one while the compressive signals show errors under $2.5 \%$, showing that all modal information of the raw signal is significantly reflected. Table V shows RE and CR of 0.4461 and 0.095 respectively, meaning $55.39 \%$ of reconstruction effectiveness and $90.5 \%$ data compression.

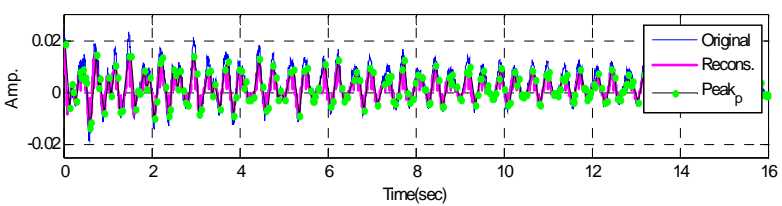

(a). Time response.

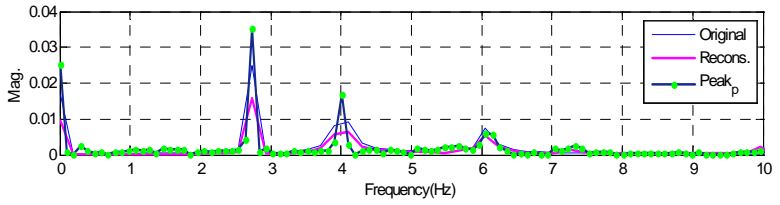

(b). Frequency response.

Fig. 8. Compare to original, reconstruction, and compressive signal.

TABLE IV: COMPARE TO EIGENVALUE OF RECON. AND PEAK-PICKING

\begin{tabular}{c|c|c|c|c|c}
\hline \hline Bending & $\begin{array}{c}\text { Wireless } \\
(\mathrm{Hz})\end{array}$ & $\begin{array}{c}\text { Recon. } \\
(\mathrm{Hz})\end{array}$ & $\begin{array}{c}\text { Error } \\
(\%)\end{array}$ & $\begin{array}{c}\text { Peak_p } \\
(\mathrm{Hz})\end{array}$ & $\begin{array}{c}\text { Error } \\
(\%)\end{array}$ \\
\hline $1^{\text {st }}$ & 2.736 & 2.736 & 0.000 & 2.735 & 0.036 \\
\hline $2^{\text {nd }}$ & 4.104 & 4.104 & 0.000 & 4.005 & 2.412 \\
\hline $3^{\text {rd }}$ & 6.058 & 6.058 & 0.000 & 6.057 & 0.016 \\
\hline \hline
\end{tabular}

TABLE V: PERFORMANCE OF AFB

\begin{tabular}{c|c|c|c}
\hline \hline RE & $\begin{array}{c}\text { Recon. } \\
\text { effect (\%) }\end{array}$ & CR & $\begin{array}{c}\text { Compressive } \\
\text { effect (\%) }\end{array}$ \\
\hline 0.4461 & 55.39 & 0.095 & 90.50 \\
\hline \hline
\end{tabular}

\section{CONCLUSION}

The purpose of this study was to compose a dynamic wireless measurement system and apply it to a model of cable-stayed bridge, in order to effectively acquire valid dynamic (acceleration) responses in real time. After evaluating the dynamic characteristics featuring in the model of cable-stayed bridge by means of the AFB for the purpose, the conclusions are as follows:

1) The dynamic wireless measurement system (DWMS) proved effective in acquiring dynamic responses of flexible structure (within $10 \mathrm{~Hz}$ ) in real-time through close congruence among its dynamic responses, those of wired measurement system and $\mathrm{FE}$ analysis.

2) The band-pass filter optimizing algorithm (BOA) of the AFB was found effective in expressing only frequency signals for the purposive mode of random signals. The effect of reconstruction was about $55 \%$, and its dynamic characteristics were found to precisely express original signals.

3) The peak-picking algorithm (PPA) of the AFB proved effective in re-sampling the picked peak-value including valid modal information of all reconstruction signals.
Especially, compression effect was about $90 \%$, and the dynamic characteristics were excellently identified only with less than $3 \%$ of error in comparison to original signals.

Dynamic characteristics of cable-stayed bridge by means of an artificial filter bank (AFB) for the purpose of efficient attainment of valid dynamic responses in real time have been determined. Therefore, this AFB system will provide new paradigm for real time measurement, data transmission, and data base management.

\section{ACKNOWLEDGMENT}

This research was supported by Basic Science Research Program through the National Research Foundation of Korea (NRF) funded by the Ministry of Education, Science and Technology (grant number: NRF-2013R1A2A1A01016192), and funded by the Ministry of Science, ICT \& Future Planning (grant number: NRF-2013R1A1A1063540).

\section{REFERENCES}

[1] M. Celebi, R. Purvis, B. Hartnagel, S. Gupta, P. Clogston, P. O. Connor, J. Yen, and M. Franke, "Seismic instrumentation of the Bill emerson memorial mississippi river bridge at cape girardeau (MO): a cooperative effort," in Proc. the 4th International Seismic Highway Conference, Memphis, Tenn, USA, pp. 29-40, 2004.

[2] M. Abe, Y. Fujino, M. Yanagihara, and M. Sato, "Monitoring of hakucho suspension bridge by ambient vibration measurement," in Proc. the Nondestructive Evaluation of Highways, Utilities, and Pipelines IV, Newport Beach, USA, March, vol. 4, no. 6, 2000.

[3] E. Y. Andersen and L. Pedersen, "Structural monitoring of the Great Belt East Bridge,” Strait crossing, pp. 189-195, 1994.

[4] C. B. Yun, J. J. Lee, S. K. Kim, and J. W. Kim, "Recent R\&D activities on structural health monitoring for civil infra-structures in Korea," KSCE Journal of Civil Engineering, vol. 7, no. 6, pp.637-651, 2003.

[5] K. Y. Wong, "Instrumentation and health monitoring of cable-supported bridges," Struct. Control Health Monit, vol. 11, no. 2, pp. 91-124, 2004.

[6] G. Heo and J. Jeon, “A smart monitoring system based on ubiquitous computing technique for infra-structural system: centering on identification of dynamic characteristics of self-anchored suspension bridge," KSCE J. Civil Engineering, vol. 13, no. 5, pp. 333-337, 2009.

[7] C. A. Peckens and J. P. Lynch, "Utilizing the cochlea as a bio-inspired compressive sensing technique," Smart Materials and Structures, vol. 22, no. $10,2013$.

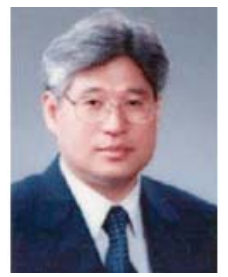

Gwanghee Heo received a $\mathrm{PhD}$ in civil engineering from the University of New Mexico in 1996. Currently, he is teaching at Konyang University. His research interests lie in structural health monitoring, vibration control, structural damage, wireless communication, earthquake engineering and so on. Recently, he is studying on a bio-inspired structural system based on bio-sensing and bio-actuating system.

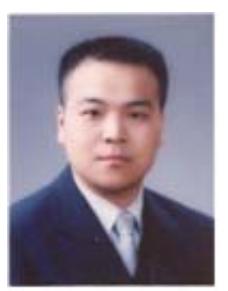

Joonryong Jeon received the BS degree in civil engineering in 2002 from the Konyang University and the MS degree in civil engineering in 2004 from the Chungnam National University. He received the $\mathrm{PhD}$ degree in civil engineering in 2011 from the Chungnam National University. Now, he is a research professor in the Department of Civil And Environmental Engineering at the Konyang University. His main research areas are smart health monitoring and vibration control of infrastructure in real-time. In addition, his research includes wireless sensing systems, design of active and semi-active dampers, and system identification techniques. 